\title{
Subphrenic and pericardial abscess secondary to unretrieved gallstone
}

\begin{abstract}
Spillage of gallstones during laparoscopic cholecystectomy is fairly common however complications from such spillage are rare. We present a rare complication of a retained gallstone after cholecystectomy, presenting with a percardial abscess and tamponade subphrenic and subhepatic abscesses, and a possible peritoneal-pericardial fistula, 2 years after cholecystectomy.
\end{abstract}

Volume 6 Issue 2 - 2018

\section{Terence Jackson, John Ammori}

Department of Surgery, Case Western Reserve University, USA

Correspondence: Terence Jackson, Department of Surgery, University Hospitals Cleveland Medical Center, Case Western Reserve University, USA, Tel 21654309 85, Email terencejamesjackson@gmail.com

\section{Case report}

$74 y / o$ male present with acute onset bilateral shoulder pain, chest pain and epigastric pain a few hours before presentation to the ED and was worse with inspiration. His past medical history is significant for coronary artery disease and his surgical history is significant for a difficult laparoscopic cholecystectomy for acute cholecystitis 2 years ago. Upon review of the operative note a comment about rupture of the gallbladder and spillage of stones was noted.

On physical exam, he was a febrile with normal vital signs and had a tender epigastrium without peritonitis. Given his cardiac comorbidities and chest pain, a coronary event was first ruled out with troponins and EKG. Other laboratory studies were significant for a WBC of 19.2. On imaging work up, his CXR showed no pneumoperitoneum and no acute cardiopulmonary processes. Given his history of upper abdominal pain and a h/o difficult laparoscopic cholecystectomy, a right upper quadrant ultrasound was obtained which showed a hypo echoic collection superior to the liver with a hyperechoic focus in it (Figure 1). A CT scan was obtained which showed a subphrenic fluid collection with a $2 \mathrm{~cm}$ stone in it, a subhepatic fluid collection and moderate pericardial effusion. There was also a concern for a pericardial-peritoneal fistula (Figure 2).

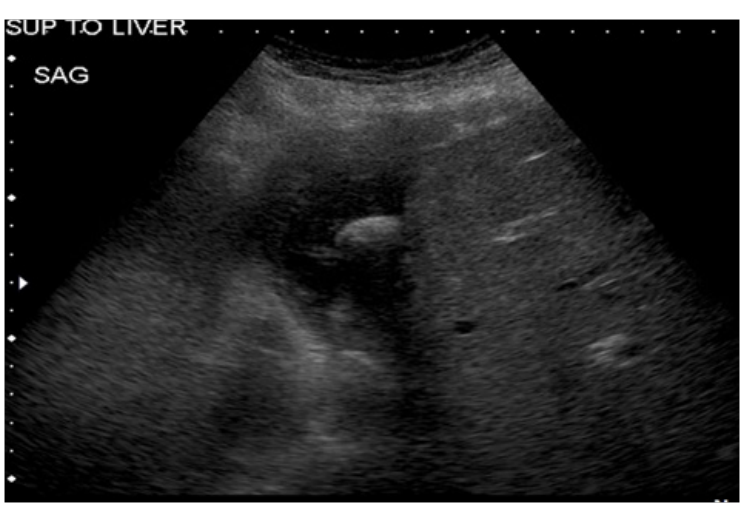

Figure I A right upper quadrant ultrasound was obtained which showed a hypo echoic collection superior to the liver with a hyperechoic focus in it.
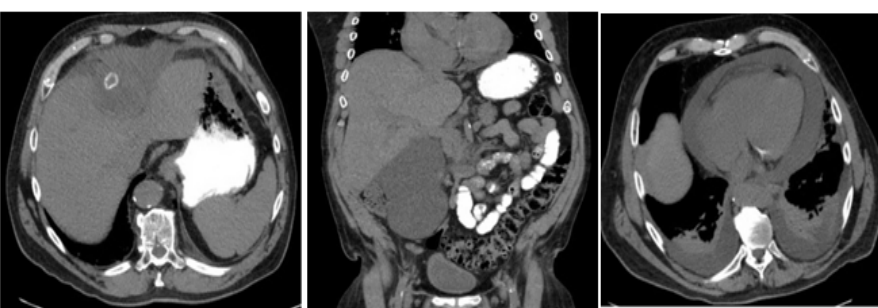

Figure 2 A CT scan was obtained which showed a subphrenic fluid collection with a $2 \mathrm{~cm}$ stone in it, a subhepatic fluid collection and moderate pericardial effusion. There was also a concern for a pericardial-peritoneal fistula.

Based on these findings, a percutaneous drainage of the intraabdominal abscesses was planned but during the procedure he had tamponade and required an emergent pericardiocentesis. Cultures from both procedures grew E. Coli. With all the available information we inferred that the stone was acting as a nidus for these abscesses and decided to perform a pericardial drainage, laparoscopic drainage of intra-abdominal abscess and retrieval of stone.

Due to concerns for a fistulous communication between the pericardial and peritoneal cavities, the procedure was begun in the chest first to reduce the chances of cardiac tamponade from pneumopericardium. A subxyphoid pericardial window was performed and a $32 \mathrm{fr}$ chest tube was placed. On laparoscopy, significant adhesions were noted above the liver (Figure 3). Dissection above the liver was challenging given the chronic inflammation and the proximity of the abscess cavity to the hepatic veins. The abscess cavity in the sub diaphragmatic space was entered and the stone was found (Figure 4). The remnant abscess was drained and a flat Jackson Pratt drain was left in the cavity after irrigation. The stone was retrieved using an end catch bag (Figure 5). The patient's postoperative recovery was uneventful. The pericardial drain was removed prior to discharge and his abdominal drain was removed in clinic. He was sent home with a 2 week course of ceftriaxone.

\section{Discussion}

Spillage of gallstones during laparoscopic cholecystectomy occurs 
in approximately $6 \%$ of cases but has been reported to be as high as $40 \%$. Complications from such spillage are very rare $(0.08-0.3 \%)$. The most common complications are infectious presenting as abdominal wall or intra-abdominal abscesses.In our review of the literature; this is the first case of a pericardial abscess/purulent pericarditis, tamponade and subphrenic abscess caused by a retained intraperitoneal abscess.

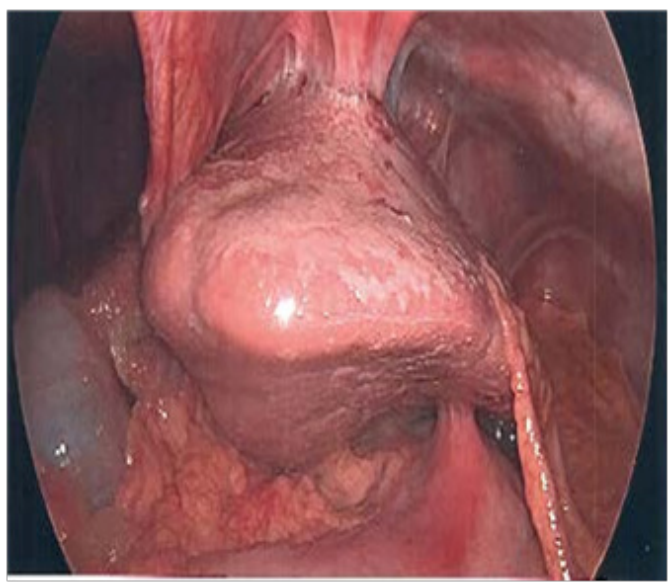

Figure 3 Significant adhesions above the liver.
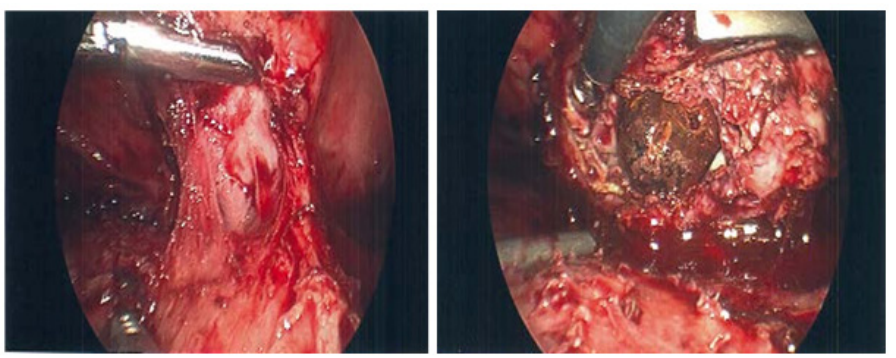

Figure 4 The abscess cavity in the sub diaphragmatic space was entered and the stone was found.

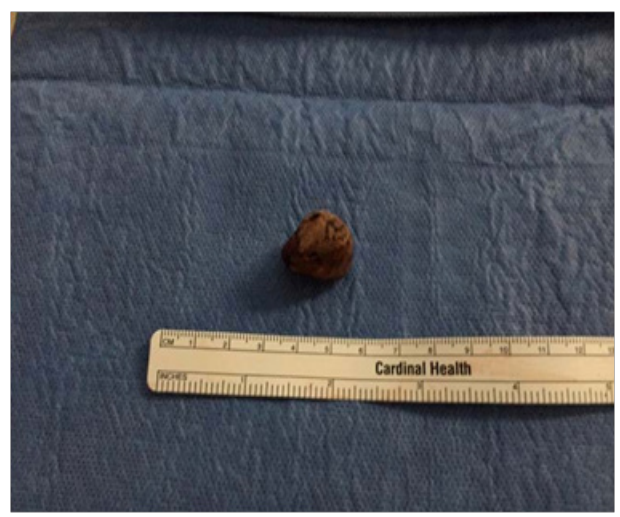

Figure $\mathbf{5}$ The stone was retrieved using an end catch bag.
Risk factors for infectious complications include larger and more numerous stones, pigmented stones and age. Some considerations during the procedure that can minimize these complications include minimizing spillage of bile and gallstones. This can be done by applying a clip or a grasper on the area of gallbladder perforation. Other considerations include focused irrigation and suction without spreading the stones further into the peritoneal cavity and careful removal of all stones using an endocatch bag. Clear documentation of spillage of bile and stones is very important to help guide future surgeons and procedures. Spillage of bile or gallstones is not an indication for conversion to laparotomy. Retrospectively, conversion to laparotomy has not shown to be of any more benefit to the patient when compared to laparoscopy. ${ }^{1-4}$

In summary, this was a case of a rare complication of a retained gallstone after cholecystectomy, presenting with a percardial abscess and tamponade, subphrenic and subhepatic abscesses, and a possible peritoneal-pericardial fistula, 2 years after cholecystectomy. As surgeons, prevention of spilled or retained stones is very important as is the recognition of retained stones and their complications.

\section{Acknowledgements}

None.

\section{Conflict of interest}

There is no conflict of interest.

\section{References}

1. Demirbas BT, Gulluoglu BM, Aktan AO. Retained abdominal gallstones after laparoscopic cholecystectomy: A systematic review. Surgical Laparoscopy, Endoscopy \& Percutaneous Techniques. 2015;97-99.

2. Schäfer M, Suter C, Klaiber C, et al. Spilled gallstones after laparoscopic cholecystectomy. A relevant problem? A retrospective analysis of 10,174 laparoscopic cholecystectomies . Surgical endoscopy. 1998;12(4):305-309.

3. Yethadka R, Shetty S, Vijayakumar A. Attitudes and Practices of Surgeons towards Spilled Gallstones during Laparoscopic Cholecystectomy: An Observational Study. Int Sch Res Not. 2014;1-5

4. Zehetner J, Shamiyeh A, Wayand W. Lost gallstones in laparoscopic cholecystectomy: all possible complications. Am J Surg 2007;193(1):73-78. 